\title{
Psychodynamic interpersonal therapy
}

\author{
Elspeth Guthrie
}

The dramatic development of cognitive-behavioural therapy (CBT) over the past 30 years, with associated high-quality research into the evaluation of its treatment effects, is to be welcomed and applauded. This form of therapy is now a recognised psychological treatment of choice for a wide variety of psychological disorders. The role of cognitive processes in the development and prolongation of psychological symptoms has been studied, and resulted in the development of coherent and effective treatment models.

In contrast, psychodynamic psychotherapies have lagged somewhat behind their younger sibling. Although theoretical thinking has continued to develop, there has been a reluctance to test the efficacy of dynamic therapies in a controlled format. Genuine concerns regarding the manualisation of therapies, the intrusiveness of objective assessments during therapy, the use of audiotaping equipment, the difficulties in measuring 'unconscious processes', and the emphasis upon symptomatic outcome as opposed to dynamic change, have all been expressed. As a result, however, there are very few controlled evaluations of dynamic psychotherapies, and both the Review of Strategic Policy in the UK (Parry \& Richardson, 1996) and the recent influential review of psychotherapy research by Roth \& Fonagy (1996), have highlighted the need for more controlled evaluations of dynamic therapies.

The relative lack of outcome research on dynamic therapies may have serious consequences. Dynamic therapies cannot be recommended as 'the treatment of choice' for particular conditions, as there is little empirical evidence to support such statements. In the USA there has been a move towards the recognition and definition of empirically supported treatments (ESTs; DuRubeis \& Crits-Christoph, 1998). These are manualised psychological therapies which have been shown to be efficacious in controlled research with a delineated population
(Chambless \& Hollon, 1998). It is possible, in the future, that therapists (in the USA) could be vulnerable to medico-legal action, if they use an unrecognised psychological treatment for a condition which has a recognised EST. Some training courses in the USA are also now only offering training in therapies that have been empirically validated.

Another adverse consequence of the lack of outcome research on dynamic therapies has been the emphasis upon cognitive processes in the development of psychological disorder, and the relative neglect of the importance of relational processes. Both, of course, are important.

Sadly, psychodynamic psychotherapies have been caricatured as being rather intense, woolly therapies that do not have scientific credibility. There is, however, increasing evidence that relational-based therapies are as efficacious as cognitive therapy and should be developed as possible alternatives to this approach. Klerman et al's (1984) interpersonal therapy has been demonstrated to be as efficacious as cognitive therapy for the treatment of depression (Elkin et al, 1989) and eating disorders (Fairburn $e t$ al, 1993). It is an efficacious treatment for recurrent depression (Franket al, 1990, 1991) and a recent study has shown that it is effective in treating depression in patients who are HIV-positive (Markowitz et al, 1998). Cognitive-analytical therapy is another relational-based therapy which is becoming increasingly popular in the UK. It has been developed by Anthony Ryle (1991), and although it has not been formally evaluated in a randomised controlled trial format, there are encouraging results from open studies (Ryle, 1995). A randomised controlled trial to evaluate cognitive-analytical therapy in the treatment of patients with borderline personality disorder is currently being planned.

This article focuses upon a particular form of psychotherapy, so called psychodynamic interpersonal therapy. Psychodynamic interpersonal

Elspeth Guthrie is Senior Lecturer in Psychological Medicine at the School of Psychiatry and Behavioural Sciences, University of Manchester (Rawnsley Building, Manchester Royal Infirmary, Oxford Road, Manchester M13 9WL). She is a liaison psychiatrist and psychoanalytical psychotherapist. 
therapy is one of the few dynamic psychotherapies which has been evaluated in several randomised controlled trials, and is an empirically supported treatment for depression. The model lies somewhere between traditional psychodynamic approaches and the interpersonal psychotherapy developed by Klerman et al (1984). Although research into the model is at a relatively early stage, the findings to date suggest that this form of psychodynamic psychotherapy has clinical validity and is a valuable treatment for depression and somatisation.

\section{The model}

Psychodynamic interpersonal therapy was developed and refined over the course of 30 years by R. F. Hobson, Reader in Psychotherapy at the University of Manchester and a former Training Analyst of the Society of Analytical Psychology in London. A training package and manual were first developed in 1983; subsequently a brief manual and rating scale for the treatment of depression (Shapiro \& Startup, 1991) were developed in 1991. A videotaped teaching package was developed by Margison \& Hobson (1983), which consists of three videotapes in which the main aspects of the model are described. The therapy is relatively easy to learn and skills are maintained over at least a two-year period (Moss et al, 1991). A book which describes the development of the model, the manual and the research conducted upon it to date, is due to be published shortly (Barkham et al, 1999).

The model is theoretically derived from psychodynamic principles, but also draws upon humanistic and interpersonal concepts. It was originally called the 'conversational model of therapy' as the main task of the therapist in this model, is to develop, with the patient, what Hobson termed a "mutual feeling language" and a relationship of "aloneness-togetherness" (Hobson, 1985).

Hobson developed the model as an attempt to move away from the traditional psychoanalytic approach of a one-sided asymmetrical relationship. He also wanted to convey to others a form of psychotherapy that was relatively free of jargon, yet had specified skills which could be learned, practised and tested.

The conversational model of psychotherapy was designed for the therapy of patients or service users whose symptoms and problems arose from difficulties or disturbances in interpersonal relationships. It is not, therefore, symptom-specific like cognitive therapy, and it can be used in its basic format to help individuals with a variety of symptomatic complaints (e.g. depression, anxiety, eating disorders). The practical working of the model can be enhanced by tailoring it to certain conditions. For instance, depression and somatisation. However, the basic underlying process is similar no matter which particular symptom complex the patient is suffering from.

The model has been conceptualised as consisting of seven different, but interlinking, components (see Box 1). Some of these are generic to all psychotherapies, but taken as a whole they constitute a specific definable model of therapy.

\section{Exploratory rationale}

One of the main aims of the therapy is to identify interpersonal difficulties in the service user's life which are responsible for either precipitating or maintaining their symptoms. The therapist tries to provide a rationale for the patient, in relation to the therapy, which emphasises the importance of linking emotional symptoms (or somatic symptoms) to interpersonal difficulties or dilemmas.

By the end of the first session or sessions, the therapist should be able to construct an interpersonal formulation which links interpersonal difficulties to the development or continuation of emotional problems and distress, which in turn may lead to further interpersonal difficulties. This formulation is shared with the patient and becomes one of the focal points for the remainder of the therapy.

\section{Shared understanding}

One of the main tasks of the therapist is to try to understand what the patient or service user is really experiencing and feeling. This is a difficult task which is probably only ever partially achieved. The following features of the model encourage the development of greater openness and understanding between the therapist and service user.

\section{Statements}

The therapist uses statements rather than questions. Questions tend to make the therapeutic situation more one-sided and may make the patient feel as if

Box 1. Main components of 'the model'

Exploratory rationale

Shared understanding

Staying with feelings

Focus on difficult feelings

Gaining insight

Sequencing interventions

Making changes 
he or she is being put on the spot and has to come up with the right answer. A statement suggests a starting point from which the patient can diverge and develop various themes. All statements are made in a tentative manner.

\section{Language of mutuality}

In psychodynamic interpersonal therapy, the therapist explicitly refers to the relationship between therapist and patient in terms of first-person words ' $I$ ' and 'we'. This indicates an active and mutual involvement in exploration. It also facilitates a deepening of the relationship between the therapist and the patient and accentuates a directness of communication.

\section{Negotiating style}

The 'how' of the therapist's talk is crucial. The therapist is really trying to say to the patient or service user: "this is how I see things now, but I might not be right, I may have misunderstood, I'd like you to help me see things more clearly". A negotiating style produces an atmosphere of collaboration between the patient and therapist where deeper understanding is reached through a series of gradual adjustments of meaning which get closer and closer to the patient's experience.

\section{Metaphor}

Use of metaphor in literature refers to the fusing of two or more images and/or ideas to bring a new experience and a new order and meaning. Metaphor is not exclusive to psychodynamic interpersonal therapy, but it is often used by therapists to bring vividness to an idea, to expand understanding of an experience or concept, and to deepen the level of emotional exchange between the patient and the therapist. The following example shows how the metaphor of a 'spring' can be used to explore a patient's feelings of tension and frustration.

Patient: "I feel on edge all the time... I just can't settle..."

Therapist: "Sounds as if you feel sort of wound up."

Patient: "I feel myself getting tighter and tighter inside... everything's rigid..."

Therapist: "... feels a bit like you feel like a spring that's all coiled up being turned tighter and tighter."

Patient: "Yeah, I think sometimes people do things deliberately to wind me up... I'm sure I'm going to just snap."

\section{Understanding hypotheses}

Hypotheses in psychodynamic interpersonal therapy are ways of promoting exploration and understanding of the patient's feelings, especially in interpersonal relationships. There are some similarities between hypotheses and interpretations, which are exploratory statements, used in other kinds of interpersonal and dynamic therapies. Hypotheses, however, are offered with much less certainty and conviction than interpretations and they are usually couched in more subtle language than interpretations, which are usually more direct.

\section{Staying with feelings (focus on 'here and now')}

This technique involves focusing on what the patient is experiencing during the interview. Instead of talking about feelings in the abstract or as if they belong only to the past, an attempt is made to recreate them or facilitate the actual expression of them in the immediacy of the therapeutic environment.

\section{Focus on difficult feelings}

'Hidden affect' refers to two situations in therapy. The first occurs when a service user is overtly expressing a particular emotion such as anger but is unaware that he or she is angry. The second situation arises when the service user is not overtly emotional, but from a rational perspective would be expected to have strong feelings.

In the first case, the therapist may address the issue of the hidden feeling by making a cue-based intervention, but contrast the service user's nonverbal and verbal cues with what he or she is actually saying. In the second situation, the service user may seem calm or disinterested when discussing something of great emotional significance. The therapist can comment upon this.

\section{Gaining insight}

Wherever possible the therapist should try to draw parallels or point out patterns in different relationships that the service user has had. This may involve making links between aspects of the service users's childhood relationships and adult relationships, or between different adult relationships.

\section{Linking hypotheses}

Linking hypotheses are statements that link feelings that have emerged in the therapy sessions to other feelings both inside and outside the therapy. They are invariably a way of drawing links between the patient-therapist relationship and other important relationships in the patient's life, past or present. In this respect, they may refer to the 
transference relationship between the patient and therapist.

\section{Explanatory hypotheses}

These statements are more complex and are only made after the therapist has acquired considerable information about the patient. They introduce the possibility of underlying reasons for problems and difficulties in relationships. They usually relate to a repeated pattern of maladaptive behaviour, both

Box 2. Example of the structure of an eightweek therapy: brief therapy (1-8 sessions)

Initial sessions

Symptom history

Staying with and exploring feelings

Nature of interpersonal relationships

Link symptom development or maintenance to interpersonal difficulties

Assessment of relationship between patient and therapist

Identification of main problem areas

Interpersonal formulation

Agreement of focus

Review of length of therapy with agreed date for ending

Intermediate sessions

Review symptoms

Rtay with feelings

Facilitate exploration of hidden feelings

Identification of factors that aggravate symptoms

Review interpersonal difficulties

Review patient's feelings regarding sessions

Exploration and linking of interpersonal difficulties to symptom formation

Linking of interpersonal difficulties between patient and therapist to interpersonal difficulties in real life

Final sessions

Explicit discussion of ending

Link ending to previous loss/dilemmas regarding intimacy

Review symptoms

Facilitate expression of negative feelings

Review insight and gains from therapy

Emphasise changes

Review how work can be continued by patient although therapy will end

Say goodbye inside and outside of therapy. The therapist tries to link the patient's behaviour to some kind of underlying conflict or difficulty. This provides an opening whereby this conflict can be acknowledged, owned and explored. It may also lead to other ways of dealing with the underlying difficulty.

\section{Sequencing of interventions}

There are many different components of the model, and it is important in the therapy that different aspects of the model are used in a coherent fashion. It is inappropriate to use an explanatory hypothesis without first establishing the emotional context and clear links to interpersonal difficulties. Before moving to understanding interpersonal difficulties, the model emphasises the importance of staying with feelings.

\section{Change}

The therapist should actively acknowledge and encourage important changes that the patient makes in therapy. The patient may be able to share feelings of sadness that he or she has not expressed before, or may be able to feel angry without getting out of control. Change may occur in the patient's relationships.

\section{Structured brief format}

Psychodynamic interpersonal therapy can be used for either brief or long-term work. When brief psychotherapy is being conducted, it is particularly important that a secure structure is established from the beginning.

Box 2 provides an example of a structure for a brief eight-week therapy. It is meant as a guide, and should not be rigidly followed. A clear contract should be established, at the start of therapy, with the number and length of sessions and a finishing date.

\section{Evaluation of psychodynamic interpersonal therapy}

Psychodynamic interpersonal therapy has been evaluated in three controlled studies as a treatment for depression and in two controlled studies for the treatment of somatisation. As one of the studies is awaiting publication, preliminary data which has been presented at research meetings will be presented where complete data are not yet available. 


\section{Treatment for depression}

There are three main studies, all of which involve a comparison of psychodynamic interpersonal therapy with CBT. They are the Sheffield Psychotherapy Project (SPP-1; Shapiro \& Firth, 1987; Box 3), the Second Sheffield Psychotherapy Project (SPP-2; Shapiro et al, 1994, 1995; Box 4) and the Collaborative Psychotherapy Project (CPP; Barkham et al, 1996; Box 5). Two were conducted in a research setting (SPP-1 and SPP-2), and involved the recruitment and treatment of white-collar workers with depression from a research clinic. These studies, as described earlier, were designed to examine explanatory models and processes of change. The other study (the CPP) was carried out in a clinical setting, to determine the extent to which the outcome results from the first two studies could be generalised to National Health Service (NHS) out-patients.

In order to be recruited to any of the three studies, patients had to have symptoms of depression above a certain level. In SPP-1 service users had to score at least 14 on the Present State Examination (PSE; Wing et al, 1974) and in SPP-2 and CPP they had to score above 16 on the Beck Depression Inventory (BDI; Beck et al, 1961). Patients in SPP-2 and the CPP were stratified according to the severity of their depressive symptoms; mild severity (BDI score 16$20)$, moderate severity (BDI score 21-26) and severe (BDI score 27+).

These studies were all conducted with methodological rigour: all service users were assessed independently by researchers not involved in delivering the therapy; all sessions were audiotaped and rated for adherence to either psychodynamic interpersonal therapy or CBT; and a variety of postsession questionnaires were administered to study aspects of the process of therapy.

The form of CBT used for comparison with psychodynamic interpersonal therapy in all the studies was termed prescriptive therapy. It was manualised and included specific features designed to address job strain, as the service users in the first two studies (SPP-1 and SPP-2) were white-collar workers, management and cognitive restructuring of work attitudes, delegation training and functional analysis and remediation of concentration and memory. The package was individually tailored to each service user and maintenance strategies were emphasised throughout.

\section{$S P P-1$}

The SPP-1 employed a cross-over design in which service users either received an eight-week course of psychodynamic interpersonal therapy followed by prescriptive therapy ( $\mathrm{a}$ form of CBT, for eight weeks) or a course of prescriptive therapy followed by psychodynamic interpersonal therapy (see Box 3 ). The main aim of this study was to determine whether an eclectic package of therapy (i.e. psychodynamic interpersonal therapy plus a cognitivebehavioural model) was an efficacious treatment for depression and to discover whether the order of delivering the two different kinds of psychotherapy made a difference to outcome.

The investigators found that there was no difference in outcome between the two trial groups, that

Box 3. SPP-1: design and main findings (Shapiro \& Firth, 1987)

Design: Cross-over study. Psychodynamic interpersonal therapy (eight weeks) + prescriptive therapy (eight weeks) $v$. prescriptive therapy (eight weeks) + psychodynamic interpersonal therapy (eight weeks).

Criteria: White-collar workers, PSE scores at least 14 . No previous experience of therapy.

Therapists: Four.

Assessments: PSE, BDI, SCL-90 and SAS. Assessments conducted at intake, mid-therapy (between treatments), end of therapy and at three-month follow-up.

Recruitment: Subjects ( $n=108)$ referred to research clinic, 55 attended for intake assessment, 48 met inclusion criteria, 40 completed treatment.

Analysis: Conducted upon treatment completers.

Results: Of the patients $65 \%$ ( $n=40)$ showed clinically significant change (i.e. end-of-treatment scores of at least 2 s.d. beyond the intake mean).

No major differences between psychodynamic interpersonal therapy and prescriptive therapy.

BDI, Beck Depression Inventory (Beck et al, 1961); PSE, Present State Examination (Wing et al, 1974); SCL-90, Symptom Checklist (Derogatis et al, 1973); SAS, Social Adjustment Scale (Weissman \& Bothwell, 1976). 
Box 4. SPP-2: design and main findings (Shapiro et al, 1994, 1995)

Design: Randomised controlled trial. Service users stratified into three groups according to BDI scores (low: 16 -20, moderate: 21-26, high: $27+$ ).

Criteria: White-collar workers, history of presenting symptoms for less than two years.

Therapists: Five; each saw six service users per psychotherapy group (two severe, two moderate and two mildly depressed).

Assessment measures: PSE, BDI, SCL-90-R, IIP (Horowitz et al, 1988) and SAS. Assessments were conducted at intake, end of therapy (8 sessions), mid-therapy (16 sessions), end of therapy (16 sessions) and at three-month and one-year follow-up.

Recruitment: 257 completed intake interview; 150 were randomised into treatment; 12 withdrew before starting treatment; 15 did not complete treatment and three did not return post-treatment questionnaires - these 18 subjects were replaced; 117 completed treatment and returned questionnaires $(50 \%$ female, $65 \%$ were married or cohabiting and the mean age was 40.5 (s.d. $=9.5$ years).

Analysis: Conducted upon treatment completers.

Results: Psychodynamic interpersonal therapy was as effective as CBT on most measures. At threemonth follow-up, patients with high BDI scores improved substantially more after 16 sessions of therapy. At one year the differential treatment effect disappeared. Sixteen-session psychodynamic interpersonal therapy was significantly better than eight-session psychodynamic interpersonal therapy and was numerically (but not statistically) significantly better than 16-session CBT. Eightsession psychodynamic interpersonal therapy was not as effective as either eight- or 16-session CBT.

SCL-90-R, Symptom Checklist (Derogatis et al, 1983); SAS, Social Adjustment Scale Self-Report (Cooper et al, 1982); BDI, Beck Depression Inventory (Beck et al, 1961); PSE, Present State Examination (Wing et al, 1974).

Box 5. CPP: design and main findings (Barkham et al, 1996)

Design: Service users were stratified into three groups according to BDI scores (low: 16-20, moderate: 21-26, high: 27+) and randomly assigned to one of two treatment approaches (psychodynamic interpersonal or CBT) of two time-limited durations (eight or 16 sessions).

Criteria: White-collar workers, continuous history of presenting disorder for less than two years, no significant change in psychotropic medication within the previous six weeks, no more than three sessions of psychotherapy within the previous five years.

Assessment measures: PSE, BDI, SCL-90-R, IIP (Horowitz et al, 1988) and SAS. Assessments conducted at intake, end of therapy (8 sessions), mid-therapy (16 sessions), end of therapy (16 sessions) and at three-month and one-year follow-up.

Recruitment: 54 met study criteria; three were withdrawn because of a therapist's illness; three dropped-out before first session; 12 dropped-out during therapy, leaving 36 completers.

Therapists: Four.

Analysis: Conducted upon treatment completers.

Results: Psychodynamic interpersonal therapy was as effective as CBT on most measures. At threemonth follow-up, patients with high BDI scores improved more substantially after 16 sessions of therapy. At one year, however, this differential treatment effect disappeared, and there was no particular advantage for the longer duration of therapy in service users with high severity scores.

SCL-90-R, Symptom Checklist (Derogatis et al, 1983); SAS, Social Adjustment Scale Self-Report (Cooper et al, 1982). BDI, Beck Depression Inventory (Beck et al, 1961); PSE, Present State Examination (Wing et al, 1974). 
is, patients who received both psychodynamic interpersonal therapy and CBT had similar outcomes, no matter the order in which they received the therapy. Both trial groups showed substantial improvements in terms of depressive symptomatology.

A slight, non-statistically significant advantage for the CBT was seen on some of the symptom measures. However, further analysis showed that this difference was attributable to the effect of one therapist in the study. Out of the four therapists involved in SPP-1, one particular therapist's service users showed significantly greater gains with prescriptive (CBT) therapy compared with psychodynamic interpersonal therapy. This difference was not shown by the other therapists. The therapist was not more effective than the other therapists overall, as that person's greater effectiveness in CBT was balanced by less good results with psychodynamic interpersonal therapy.

These findings highlight the importance of differential therapist effectiveness when considering psychotherapy outcome studies. The two therapies in this study were manualised, and independent ratings confirmed that the therapists adhered to the different treatment modalities. Treatment manuals help to standardise therapeutic interventions, but they do not eliminate an individual therapist's affinity for one kind of therapeutic approach compared with another.

\section{SPP-2}

In SPP-2, as with SPP-1, there was very little difference in terms of outcome between psychodynamic interpersonal therapy and CBT. Broadly equivalent outcomes of CBT and psychodynamic interpersonal therapy were found on most measures. Overall the service users who received either psychodynamic interpersonal therapy or CBT in this project showed substantial improvement. The pre-/post-effect sizes for the BDI and SCL-90-R-GSI (Derogatis, 1983) were 1.77 and 1.35 , respectively. The overall pre-/ post-IIP-effect size was 1.00 .

Service users who received psychodynamic interpersonal therapy in either an eight-session or sixteen-session format showed substantial improvement in terms of their depressive symptomatology, however, sixteen sessions produced greater overall long-term benefit than eight sessions of psychodynamic interpersonal therapy. Adjusted mean BDI scores at 12-month follow-up, were 5.21 for patients receiving sixteen sessions, and 11.39 after eight sessions $(F(1,45)=5.19, P=0.03)$. For those service users with high intake scores (over 14 on the BDI), 53 out of $90(59 \%)$ showed clinically significant change at the end of eight sessions of psychodynamic interpersonal therapy, and 58 out of $80(72 \%)$ at the end of the sixteen-session treatment. Although service users did show improvement following eight sessions of psychodynamic interpersonal therapy, it did not perform as well as the other three treatments in the study (sixteen- and eight-session CBT, and sixteen-session psychodynamic interpersonal therapy).

At one-year follow-up, there were no main effects of treatment on any measure. On the BDI, those who had received psychodynamic interpersonal therapy and CBT returned adjusted means of 8.25 and 7.15, respectively $(F<1)$.

Examination of dose-response curves suggested that symptomatology improved in a linear fashion over time. Thus, the longer the therapy, the greater the improvement.

This study confirmed that psychodynamic interpersonal therapy is an efficacious treatment for depression. There was very little difference in treatment effect between psychodynamic interpersonal therapy and CBT, particularly at the one-year follow-up; both resulted in substantial improvements. Sixteen sessions of therapy were superior to eight sessions of treatment, particularly for patients with more severe symptoms.

\section{CPP}

This study was designed to test whether the results of the SPP-2 could be replicated in a clinical setting. The main design and results of the study are shown in Box 5. As in SPP-2, all subjects in the study were suffering from depression, but they were all recruited from NHS clinics, instead of from research clinics.

The findings from this study, suggested that patients who received either psychodynamic interpersonal therapy or CBT showed substantial improvement in their symptoms of depression. However, the results were not as striking as in the SPP-2. Although the initial treatment response was similar to that found in SPP-2, service users treated in the NHS setting (CPP) did not maintain their improvement, over the 12-month follow-up period, as well as service users treated during the SPP-2 study. There was a greater trend for their symptoms to recur.

It is not surprising that the results in CPP were not as impressive as in SPP-2, as most therapies tested in a research setting, with highly selected service users, do not appear to perform as well in a clinical environment. The findings from the CPP study demonstrated that psychodynamic interpersonal therapy can be delivered in a busy NHS setting. It was acceptable to service users, and the majority (75\%) completed treatment with improvement in their symptoms of depression. It is possible, however, than some patients in a clinical setting may need 
further treatment during the following 12 months to prevent relapse.

\section{Psychodynamic interpersonal depression studies}

The considerable body of work from Shapiro et al, which has been conducted over the past 10 years, has confirmed that psychodynamic interpersonal therapy is an effective treatment for depression and can be employed in a clinical NHS setting. It appears to be equivalent in its effect to CBT.

The therapists in all the above studies did not hold any particular allegiance to either psychodynamic interpersonal therapy or CBT, however, it is important to note that they all had a background training in CBT, and none had had a dynamic training. This may explain why one of therapists in SPP-1 and SPP-2 was particularly good at CBT, and less successful using a psychodynamic interpersonal approach. However, the results suggest that psychodynamic interpersonal therapy can be used effectively by therapists who do not have a background in dynamic psychotherapy.

\section{Studies on patients with somatic symptoms}

Psychodynamic interpersonal therapy has been evaluated as treatment for somatisation in two studies involving patients with functional gastrointestinal disorders. The first study involved patients with irritable bowel syndrome (Guthrie et al, 1991; Box 6) and the second study (Box 7) involved patients with functional dyspepsia (futher details available from the author upon request). Functional gastrointestinal disorders are common conditions. Patients with such problems describe symptoms for which there is no identifiable organic gastro-intestinal disorder. Irritable bowel syndrome is characterised by abdominal pain, abdominal distension and an altered bowel habit, whereas the key feature of functional dyspepsia is upper abdominal pain. There is a great deal of overlap between these conditions and the other functional bowel disorders.

Individuals in the community with either irritable bowel syndrome-like symptoms or symptoms of functional dyspepsia have similar rates of psychological disturbance to community control subjects. Seeking treatment, however, is associated with an increased likelihood of psychiatric disorder, and $40-50 \%$ of patients seen in gastro-intestinal clinics with irritable bowel syndrome have a psychiatric disorder.

Patients in both of the psychotherapy trials were selected for symptom chronicity, and represent individuals at the extreme end of the spectrum of patients with somatic symptoms. The primary aim of both studies was to assess the effectiveness of psychodynamic interpersonal therapy in a naturalist setting, so both included a control condition and carried out 'intention-to-treat' as well as 'treatmentcompleter analyses'. Preliminary data, presented at scientific meetings, will be presented on the latter project as full details await publication.

Psychodynamic interpersonal therapy in patients with irritable bowel syndrome

In this first study, brief psychodynamic interpersonal therapy was compared with a placebo control condition, in patients with chronic and intractable irritable bowel syndrome (Guthrie et al, 1991). Patients meeting the study criteria were recruited consecutively from one gastro-intestinal clinic in a large teaching hospital. The patients were therefore representative of subjects with irritable bowel syndrome with chronic and enduring symptoms, but are not representative of most patients with irritable bowel syndrome who have less severe problems.

Psychodynamic interpersonal therapy was specially adapted for the treatment of patients with somatisation. A long first session lasting 3-4 hours was used to engage patients in the treatment. This was followed by six 'normal length sessions'. The placebo control condition consisted of supportive listening, where patients saw a therapist who was supportive and listened to their problems, but did not make any specific interventions. Patients in the control condition did not receive a long first session, and all sessions were of $\mathbf{3 0}$ minutes duration.

The study had a partial cross-over design. Following recruitment, patients were randomly assigned to either psychodynamic interpersonal therapy or the control condition. At the end of the trial period, patients in the control group whose symptoms had not improved were offered psychodynamic interpersonal therapy. Outcome was independently assessed by gastroenterologists who remained blind to the trial groups.

Box 6 shows the main results of the study. Patients who received psychodynamic interpersonal therapy reported significant improvements in both psychological and physical symptoms in comparison to controls. Patients in the control group, showed a small improvement during the control condition, followed by a much greater improvement in their symptoms after they had received psychodynamic interpersonal therapy. Patients who received therapy maintained their improvement at 12-month followup. Patients who dropped out of the study (either from the treatment group or the control group) 
Box 6. A controlled trial of psychological treatment for irritable bowel syndrome (Guthrie et al, 1991).

Design: Randomised controlled trial. Control subjects offered treatment at the end of the study.

Criteria: Irritable bowel syndrome, continuous symptoms for 12 months or more, medical treatment in a secondary setting for at least six months without improvement.

Therapist: One therapist conducted both treatment and control conditions.

Assessment measures: Bowel symptom scale, daily bowel diary, PSE, BDI, CAS. Assessments conducted at intake, end of therapy ( eight sessions), after control subjects had therapy and one year later. Self-report ratings plus independent rating from gastroenterologist blind to the trial groups.

Recruitment: 113 elligible, four refusals, five suicidal ideation, two did not speak English, two had an organic pathology; 102 subjects entered study; 77 female, median age 47 years (range 20-75)

Analysis: Conducted upon intention-to-treat and treatment completers.

Results: Psychodynamic interpersonal therapy resulted in significantly greater reductions in both bowel symptoms and psychological symptoms in comparison with control condition. Improvement sustained at one year. Thirteen patients who dropped out of the study continued to suffer from severe symptoms.

HDRS, Hamilton Depression Rating Scale (Hamilton, 1967); CAS, Clincial Anxiety Scale (Snaith et $a l, 1982) ;$ BDI, Beck Depression Inventory (Beck et al, 1961); PSE, Present State Examination (Wing et al, 1974).

continued to experience severe symptoms which did not respond to any further kind of intervention.

The best predictors of a good outcome following psychodynamic interpersonal therapy were: presence of anxiety and depression at intake, absence of constant pain, relatively short duration of symptoms and few sites of abdominal pain.
Psychodynamic interpersonal therapy in patients with functional dyspepsia

In the study on functional dyspepsia (further details available from the author upon request), the efficacy of psychodynamic interpersonal psychotherapy was tested against a supportive psychotherapy in

Box 7. A randomised controlled trial of psychotherapy in patients with chronic symptoms of functional dyspepsia (further details available from the author upon request)

Design: Randomised controlled trial.

Criteria: Functional dyspepsia, chronic symptoms, unresponsive to at least two different medical treatments

Therapists: One therapist conducted both therapies.

Assessment measures: Diary of dyspeptic symptoms, PSE, HAD, SCL-90-R, IIP, SF-36 (Ware \& Sherbourne, 1992). Assessments conducted at intake, end of therapy (12 weeks) and one year later. Patient self-report rating of bowel symptoms. Independent rating of symptoms by gastroenterologist blind to the study groups.

Recruitment: 95 elligible, 75 entered study, two were withdrawn because of organic pathology; 31 males. Mean age 40 years. Mean length of dyspeptic symptoms 33.4 months

Analysis: Conducted upon 'intention-to-treat' and on 'treatment completers'.

Results: At end of study, patients who received psychodynamic interpersonal therapy showed significantly greater improvement in dyspeptic symptoms than control subjects.

SCL-90-R, Symptom Checklist (Derogatis, 1983); IIP, Inventory of Interpersonal Problems (Horowitz et al, 1988); HAD, Hospital Anxiety and Depression Scale (Zigmond \& Snaith, 1983); PSE, Present State Examination (Wing et al, 1974). 
patients with chronic symptoms. Unlike the study on irritable bowel syndrome, the control group received an active, but low-grade treatment. As it had already been established that psychodynamic interpersonal therapy was superior to a psychological placebo in patients with chronic functional bowel disorders, it was now tested against a lowgrade, less intense and less expensive form of psychotherapy - supportive psychotherapy. Both trial groups received a long first session followed by six standard length sessions.

Preliminary data from the study on functional dyspepsia has shown that patients who received psychodynamic interpersonal therapy experienced a significantly greater reduction in dyspeptic symptoms than patients who received supportive psychotherapy (further details available from the author upon request). The gastroenterologist's independent ratings of symptom scores showed a mean (s.d.) reduction in symptoms for the psychotherapy patients of $3.36(0.73)$ compared with 0.44 $(0.65)$ for controls, $(P=005)$. The 12-month followup analysis is currently been conducted.

These studies suggest that psychodynamic interpersonal therapy is an effective treatment for patients with chronic functional bowel disorder. The therapy used to treat such patients is relatively brief, and is acceptable to most patients. A small subgroup of patients (approximately 10-15\%) cannot be helped by brief psychodynamic interpersonal therapy and may require more intensive and alternative treatment approaches.

\section{Conclusions}

There is preliminary evidence that psychodynamic interpersonal therapy is an efficacious treatment for depressive disorders and somatisation. It is relatively easy to learn, and can be practised by therapists from a wide range of different theoretical backgrounds, although it is preferable that therapists have a background training in either interpersonal therapy or dynamic therapy. It lies between dynamic psychotherapies and interpersonal therapy in its treatment approach. It may be a useful alternative to CBT, particularly, if patients or service users have prominent interpersonal dilemmas or difficulties. The model requires further evaluation in a wider range of psychological disorders, and the author is aware of five further randomised controlled trials of psychodynamic interpersonal therapy which are currently in progress. The collaborative psychotherapy project and the studies on somatisation, which all recruited clinically representative groups of patients, suggest that psychodynamic interpersonal therapy is a treatment that is relevant to NHS practice. In the clinical setting, not all patients respond to CBT, and psychodynamic interpersonal therapy or other relational therapies may be useful.

\section{References}

Barkham, M., Rees, A., Shapiro, D. A., et al (1996) Outcome of time-limited psychotherapy in applied settings: replicating the Second Sheffield Psychotherapy Project. Journal of Consulting and Clinical Psychology, 64, 1079-1085. - Guthrie, E., Hardy, G., et al (1999) PsychodynamicInterpersonal Therapy. Thousand Oaks, CA: Sage, in press.

Beck, A. T., Ward, C. H. \& Mendelson, M. (1961) An inventory for measuring depression. Archives of General Psychiatry, 4, 561-571.

Chambless, D. L. \& Hollon, S. D. (1998) Defining empirically supported therapies. Journal of Consulting and Clinical Psychology, 66, 7-18.

Cooper, P., Osborn, M., Gath, D., et al (1982) Evaluation of a modified self-report measure of social adjustment. British Journal of Psychiatry, 141, 68-75.

Derogatis, L. R. (1983) SCL-90-R: Administration, Scoring and Procedures Manual. Townson, MD: Clinical Psychometric Research.

-, Lipman, R. S. \& Covi, L. (1973) SCL-90: an outpatient psychiatric rating scale - preliminary report. Psychopharmacology Bulletin, 9, 13-28.

DeRubeis, R. J. \& Crits-Christoph, P. (1998) Empirically supported individual and group psychological treatments for adult mental disorders. Journal of Consulting and Clinical Psychology, 66, 37-52.

Elkin, I., Shea, T. M., Watkins, J. T., et al (1989) National Institute of mental health Treatment of Depression Collaborative Research program: general effectiveness of treatments. Archives of General Psychiatry, 46, 971-982.

Fairburn, C. G., Jones, R., Peveler, R. C., et al (1993) Psychotherapy and bulimia nervosa. Archives of General Psychiatry, 50, 419-428.

Frank, E., Kupfer, D. J., Perel, J. M., et al (1990) Three-year outcomes for maintenance therapies in recurrent depression. Archives of General Psychiatry, 47, 1093-1099

-, - Wagner, E. F., et al (1991) Efficacy of interpersonal psychotherapy as a maintenance treatment of recurrent depression. Archives of General Psychiatry, 48, 1053-1059.

Guthrie, E., Creed, F., Dawson, D., et al (1991) A controlled trial of psychological treatment for the irritable bowel syndrome. Gastroenterology, 100, 450-457.

Hamilton, M. (1967) Development of a rating scale for primary depressive illness. British Journal of Social and Clinical Psychology, 6, 278-296.

Hobson, R. F. (1985) Forms of Feeling. London: Tavistock

Horowitz, L. M., Rosemberg, S. E., Baer, B. A., et al (1988) Inventory of interpersonal problems: psychometric properties and clinical applications. Journal of Consulting and Clinical Psychology, 56, 885-892.

Klerman, G. L., Weissman, M. M., Rounsaville, B. J., et al (1984) Interpersonal Psychotherapy of Depression. New York: Basic Books.

Markowitz, J. C., Kocsis, J. H., Fishman, B., et al (1998) Treatment of depressive symptoms in human immunodeficiency virus-positive patients. Archives of General Psychiatry, 55, 452-457.

Margison, F. \& Hobson, R. F. (1983) A Conversational Model of Psychotherapy (videotapes). London: Tavistock.

Moss, S., Margison, F. \& Godbert, K. (1991) The maintenance of psychotherapy skill acquisition: A 2-year follow-up. British Journal of Medical Psychology, 64, 233-236.

Parry, G. \& Richardson, A. (1996) NHS Psychotherapy Services in England: Review of Strategic Policy. London: Department of Health. 
Roth, A. \& Fonagy, P. (1996) What Works for Whom?: A Critical Review of Psychotherapy Research. London: Guildford Press.

Ryle, A. (1991) Cognitive-Analytic Therapy: Active Participation in Change. A New Integration in Brief Psychotherapy. London: John Wiley \& Sons.

- (1995) Research relating to CAT. In Cognitive-Analytic Therapy: Developments in Theory and Practice (ed. A. Ryle), pp. 175-191. London: John Wiley \& Sons.

Shapiro, D. A., Barkham, M., Rees, A., et al (1994) Effects of treatment duration and severity of depression on the effectiveness of cognitive-behavioural and psychodynamic-interpersonal psychotherapy. Journal of Consulting and Clinical Psychology, 62, 522-534.

-, Rees, A., Barkham, M., et al (1995) Effects of treatment duration and severity of depression on the maintenance of gains following cognitive-behavioural and psychodynamic interpersonal psychotherapy. Journal of Consulting and Clinical Psychology, 63, 378-387.

Shapiro, D. \& Firth, J. (1987) Prescriptive $v$. exploratory psychotherapy: outcomes of the Sheffield Psychotherapy project. British Journal of Psychiatry, 151, 790-799.

- \& Startup, M. J. (1991) Raters' Manual for the Sheffield Psychotherapy Rating Scale. Memo no. 1154. Leeds: Psychological Therapies Research Centre, University of Leeds.

Snaith, R. P., Baugh, S. J., Clayden, A. D., et al (1982) The Clinical Anxiety Scale: an instrument derived from the Hamilton Anxiety Scale. British Journal of Psychiatry, 141, 518-523.

Ware, J. E. \& Sherbourne, C. D. (1992) The Mos 36-item short-form health survey (SF-36). 1: Conceptual framework and item selection. Medical Care, 30, 473-483.

Weissman, M. M. \& Bothwell, S. (1976) Assessment of social adjustment by patient self report. Archives of General Psychiatry, 33, 1111-1115.

Wing, J. K., Cooper, J. E. \& Sartorius, N. (1974) Measurement and Classification of Psychiatric Symptoms: an Instruction Manual for the PSE and Catego Program. London: Cambridge University Press.

Zigmond, A. S. \& Snaith, R. P. (1983) The Hospital Anxiety and Depression Scale. Acta Psychiatrica Scandinavica, 67, 361-370.

\section{Multiple choice questions}

1. The following are key components of psychodynamic-interpersonal therapy:
a staying with feelings
b focusing on negative cognitions
c shared understanding
d exploratory rationale
e mutative interpretation.

2. Psychodynamic interpersonal therapy is a psychological treatment:

a which was developed by R. F. Hobson

$b$ where the main vehicle for change is the patient-therapist relationship

c where the therapist uses statements as opposed to questions

d where the therapist never refers directly to the patient-therapist relationship

e where metaphor is used to help amplify and develop feelings.
3. Psychodynamic interpersonal therapy in the treatment of depression:

a has equivalent effects to CBT

$b$ produces the best results with an eight-session format as opposed to 16 sessions

c produces improvement over time in a linear fashion

$d$ is only effective if therapists have had a background training in dynamic psychotherapy

e results in clinically significant improvement in three-quarters of service users.

4. In patients with chronic symptoms of the irritable bowel syndrome:

a psychodynamic interpersonal therapy has been used successfully as a treatment

b a long first session lasting 3-4 hours is used to engage patients in psychodynamicinterpersonal therapy

c those with overt symptoms of depression and anxiety respond well to psychodynamic interpersonal therapy

d psychodynamic interpersonal therapy is an unacceptable treatment which most patients reject

e treatment effects of psychodynamicinterpersonal therapy are maintained for at least one year after treatment.

5. Psychodynamic interpersonal therapy:

a is relatively easy to teach and learn

$b$ is a manualised therapy

c lies between traditional dynamic psychotherapies and interpersonal therapy in its approach

$\mathrm{d}$ is a recognised treatment for a wide range of psychiatric disorders

e can only be used in a long-term format.

\section{MCQ answers}

$\begin{array}{llllllllll}\text { 1 } & & 2 & & 3 & & 4 & & 5 & \\ \text { a } & \text { T } & \text { a } & \text { T } & \text { a } & \text { T } & \text { a } & \text { T } & \text { a } & \text { T } \\ \text { b } & \text { F } & \text { b } & \text { T } & \text { b } & \text { F } & \text { b } & \text { T } & \text { b } & \text { T } \\ \text { c } & \text { T } & \text { c } & \text { T } & \text { c } & \text { T } & \text { c } & \text { T } & \text { c } & \text { T } \\ \text { d } & \text { T } & \text { d } & \text { F } & \text { d } & \text { F } & \text { d } & \text { F } & \text { d } & \text { F } \\ \text { e } & \text { F } & \text { e } & \text { T } & \text { e } & \text { T } & \text { e } & \text { T } & \text { e } & \text { F }\end{array}$

